\title{
Pesquisa em franchising: uma análise bibliométrica
}

O presente artigo tem por objetivo de pesquisa realizar uma análise dos principais avanços e tendências sobre o tema "franchising", com a finalidade de identificar autores, estudo da evolução das citações, instituições, periódicos, volume de publicações sobre o tema, artigos mais relevantes e as suas principais contribuições. O termo "franchising", foi utilizado na busca na base de dados do Web of Science por meio da análise bibliométrica que possibilitou identificar 1101 artigos. Com essa análise foi possível traçar a evolução histórica sobre o tema, bem como a análise dos picos e as baixas nas publicações e, principalmente, a ampliação do escopo de pesquisa. No início os interesses dos pesquisadores concentravam-se apenas na perspectiva econômica, envolvia relações contratuais, propriedade e distribuição, entretanto, o crescimento do modelo que favoreceu o surgimento de novas e redes e o amadurecimento de outras, fator que levou os pesquisadores a ampliar o escopo das análises, envolvendo, tecnologia, empreendedorismo, varejo, comportamento organizacional, ou seja, sobre a perspectiva de marketing, estratégias e estudos organizacionais. As mudanças não ocorrem apenas nas áreas de interesse, novos países, centros de pesquisa e novos periódicos passaram a publicar sobre o tema.

Palavras-chave: Franchising; Análise bibliométrica; Web of science.

\section{Research in franchising: a bibliometric analysis}

This article aims to carry out a research analysis of the main developments and trends on franchising research field, in order to identify the more prolific authors, the study of the evolution of the citations, institutions or universities, journals, and volume of publications on the topic, most relevant articles and their major contributions. The term "franchising" was used in the search on the Web of Science database through bibliometric analysis that enabled identify 1101 articles. With this analysis it was possible to trace the historical evolution on the topic, as well as analysis of the peaks and lows in publications and, particularly, the expansion of the search scope. At first the interests of researchers focused only on the economic perspective, involving contractual relations, property and distribution, however, the growth model that favored the emergence of new networks and the ripening of other, a factor that led researchers to expand scope of the analysis, involving technology, entrepreneurship, retail, organizational behavior, that is, from the perspective of marketing, strategy and organizational studies. The changes not only occur in the areas of interest, new countries, research centers and new periodicals began to publish on the topic.

Keywords: Franchising; Bibliometric analysis; Web of science.

\section{Topic: Empreendedorismo}

Reviewed anonymously in the process of blind peer

Belmiro do Nascimento João

Pontifícia Universidade Católica de São Paulo, Brasil http://lattes.cnpq.br/5455434263444485

belmirojoao@gmail.com

\section{Amalia Costa Farias}

Pontifícia Universidade Católica de São Paulo, Brasil http://lattes.cnpq.br/3805636940894782 amalia.farias10@hotmail.com
Received: 17/01/2017

Approved: 17/03/2017
Referencing this:

JOÃO, B. N.; FARIAS, A. C.. Pesquisa em franchising: uma análise bibliométrica. Revista Brasileira de Administração Científica, v.8, n.1, p.157-175, 2017. DOI: http://doi.org/10.6008/SPC2179684X.2017.001.0013 


\section{INTRODUÇÃO}

O sistema de franquias brasileiro obteve um crescimento significativo nos últimos anos conforme dados ABF (Associação Brasileira de Franchising), atualmente o setor movimenta $R \$ 139,593 \mathrm{bi}$; em 2005, o setor movimentava R\$35bi. Entre 2005 a 2012, o crescimento do setor foi acima de 13\% anuais, chegando a atingir a casa dos $20,4 \%$ em 2010, sendo que na época o PIB Brasil estava em 7,5\%. Apesar da queda significativa em 2013 , passando de 16,2 para $11,9 \%$, os resultados do setor apresentam-se de forma satisfatória em relação a outros segmentos e ao PIB (Produto Interno Bruto). Desde 2013, o setor vem enfrentando quedas significativas no crescimento, 11,9\% em 2013, 7,7 em 2014 e 8,3\% em 2015. No entanto, os números atuais são positivos, superando o crescimento 2003 , que atingiu a $3,7 \%$, um dos piores resultados de sistema de franchising (ABF, 2013, 2014, 2015).

As oscilações apresentadas parecem não ter afetado o setor, o número de novas redes e novos franqueados aumentam a cada ano conforme dados da ABF. O setor é considerado pelos institutos especializados como um sistema vantajoso, por ser tratar de uma transação que envolve, por um lado, o franqueador que busca expansão dos negócios e oferece o know how e, pelo outro lado, o empreendedor na busca de um empreendimento com menor risco. Apesar da instabilidade econômica e política brasileira, os especialistas afirmam que o setor tende a manter o crescimento em torno de $6 \%$ a $8 \%$ em virtude dos novos formatos ofertados.

As primeiras franquias surgiram em 1851 nos Estados Unidos, implantadas pela I. M. Singer \& Co., fabricante de máquinas de costura; porém, o sistema só passou a licenciar o direito de uso da marca em 1862. Apesar de ter iniciado no século anterior, a popularização do modelo ocorreu somente após a 2o Guerra Mundial. Em virtude do grande de número de franquias na ocasião, inicia a corrida para regulamentar o sistema de franchising, uma das leis promulgadas foi a Lei de Marcas de 1946, que possibilitou a expansão de várias das redes naquele país, entre elas: McDonald's e Burger King (FRIEDHEIM, 2013).

No Brasil, as primeiras franquias surgiram na década de 60, com o ensino de línguas, Yázigi em 1963 e a CCAA em 1969. O modelo passou a crescer no Brasil no final da década de 70, com abertura da primeira franquia do McDonald's no país, o sistema de franchising se popularizou com a abertura de novas redes, a criação da Associação Brasileira de Franchising na década de 80 e a promulgação da Lei de Franquia ( $n$-8.955/94) em 15 de dezembro de 1994 (FRIEDHEIM, 2013). No mesmo período verifica-se um esforço das redes americanas na busca de crescimento por meio de desenvolvimento de novos mercados (ROCHA et al., 2014).

Entendendo a relevância do tema que continua em adaptações e despertando interesses nos dias atuais, o presente artigo tem objetivo identificar lacunas relacionadas aos desafios enfrentados na gestão do sistema de franchising. Este estudo será realizado por meio da bibliometria que visa analisar a produção científica, bem como os indicadores que retratam o grau de desenvolvimento de uma área do conhecimento de um campo científico ou de saber (ARAÚJO et al., 2011). Dessa forma, o estudo tem o objetivo de identificar as principais temáticas, tendências e lacunas sobre o Sistema de Franchising e, adicionalmente, pretende identificar autores e suas obras mais relevantes que contribuem para o avanço das pesquisas. 
A abordagem metodológica pautou-se em um estudo bibliométrico na base de dados Web of Science de artigos localizados por meio da palavra chave "Franchising", os artigos identificados foram 1101, levando em consideração, a identificação dos principais autores, período das publicações, estudo da evolução das citações, principais journals, países que mais publicam, relações das universidades e das áreas que mais possuem publicações sobre o tema e, por fim, a identificação por meio de nuvem de palavras dos termos mais utilizados nos abstracts e das palavras-chaves dadas pelos autores. Nas seções a seguir prosseguimos com uma revisão teórica sobre o Sistema de Franchising, a metodologia utilizada, resultados da análise extraídas da base do Web of Science e as considerações finais, incluindo as lacunas identificadas para a agenda de pesquisas futuras.

\section{REVISÃO TEÓRICA}

O sistema de franchising não se limita apenas um instrumento contratual entre duas partes, mais representa um modelo de negócio formatado pelo franqueador, que vai além do contrato. É formada uma rede de parceria que trabalha para expandir a clientela e os negócios relacionados à marca do franqueador.

O sistema prevê a formalização, viabilizando o funcionamento do sistema envolvendo empresas independentes por meio de contrato entre o franqueado recebe os direitos do franqueador para usufruir da marca por determinado período de tempo (LAFONTAINE, 1992). A franquia empresarial ou franchising é a possibilidade de expandir o negócio a partir de recursos do candidato a franqueado (investidor). Os recursos envolvidos na transação não são só financeiros, envolve know how do franqueado, o seu conhecimento de mercado e a sua capacidade de gestão (ANGELO, et al., 2012).

O sistema de franchising é um modelo, ou formato, de gestão mercadológica, definida como um sistema vertical de distribuição, na qual a franquia, representada por um contrato legal, pelo qual, o franqueador concede uma licença autorizando a outra parte, franqueadora, a comercializar um produto com marca registrada, enquanto uma parte recebe o direito de vender a outra autoriza, ou seja, concede a licença (ROSENBLOOM, 2015). Para discutir o compartilhamento de risco, a padronização, relacionamento franqueado-franqueador, por isso, entende que essa relação é um problema de agência porque os contratos de franchising são formas de controlar e supervisionar com intuito de evitar conflitos entre os agentes, permitindo que cada um dos agentes cumpra o que foi estabelecido (RUBIM, 1978; BRICKLEY et al., 1987).

O contrato de franchising difere de uma firma para outra, dependendo do formato de franquia ou a estruturação da rede serve também como meio de optar por multiformatos (COMBS et al., 2004). O contrato é um documento que formaliza a padronização da franquia, manual operacional da empresa que garante a relação entre os agentes. Mesmo se tratando de uma relação contratual, a relação entre franqueador e franqueado amadurece e muda de estágio, o tempo de existência da rede, o tempo de relação com franqueado, às relações estabelecidas no contrato tendem a sofrer mudanças, que podem afetar a forma de gerenciamento da rede (DANT et al., 2003).

Portanto, é comum as redes de franchising desenvolverem um modelo de negócio, chamado Business Format Franchising, arranjo organizacional que permite a expansão por meio da distribuição 
verticalizada de produtos e serviços (ALBUQUERQUE et al., 1996). O modelo ou formato de negócio é necessário para a criação de uma proposta de valor que viabilize soluções eficazes e convenientes e acessíveis ao sistema de franchising e possibilite que seja visto como uma modalidade de gestão de negócios denominada estratégia de desenvolvimento de mercado.

O sistema passou por uma evolução de modelos, com licenciamento de marca que integra apoio operacional, tecnologia e a participação dos franqueados nas decisões e responsabilidade social. Mas nem sempre o sistema de franchising foi assim, passando por uma evolução (CHERTO et al., 2006). Os negócios mudam ao longo do tempo visando o desempenho, neste processo de mudança, as menos eficientes são substituídas e as com melhores desempenhos copiadas (KARPLAN, 2000). Desde o início da expansão do sistema de franchising nos Estados Unidos, após a segunda guerra mundial, o franchising não possuía legislação especifica e suporte ao franqueado. O setor evoluiu para atender as regulamentações implementadas pelo setor (MELO, 2012; CHERTO et al., 2006).

A evolução se deu na seguinte ordem: Trade and Product Franchising (Franquia de Produto/Marca): sem exclusividade. $O$ franqueado recebe a licença restrita ao uso da marca com suporte para a distribuição do produto, sem suporte operacional; Trade and Product Franchising (Franquia simples de produto ou marca exclusiva): o franqueado recebe a licença de uso da marca e/ou distribuição do produto, com suporte operacional; Business Format Franchising (Franquia de negócio formatado ou exclusiva): o franqueado recebe a licença de uso da marca e/ou distribuição do produto. Com suporte total, operacional e estratégico, controle total da franqueadora; Learning Network Franchising - participação dos franqueados nas decisões estratégicas através do conselho de franqueados; Rede Inteligente ou rede operacional - integração das redes por meio da informática; e Franquia Sustentável - visão de negócio rentável, justo e sustentável.

O sistema de franchising passou por evolução que refletiu em novos arranjos organizacionais, por isso, no entanto, a evolução dos formatos não aconteceram de forma homogênea, ou seja, há franquias transitando em todos os seis estágios. Portanto, ao analisar-se uma rede de franquias, aconselha-se a classificá-la, identificando qual o tipo de geração a que a rede pertence ou o formato ofertado em questão. Uma forma de verificar tal enquadramento ou categoria pertencente é fazer uma análise da COF (Circular de Franquias).

Portanto, os modelos de franchising diferem de uma franquia para outra, tudo depende do modelo de negócio descrito na circular de oferta de franquia, a COF, trata-se do primeiro documento entregue ao franqueado ao final do processo de seleção, quando o franqueador e franqueado cumprirem todas as etapas, tal documento disponibiliza informações consolidadas da situação da franquia servindo de tomada de decisão por parte do franqueado (PRADO, 2012).

O sistema muda para se adaptar ao ambiente e acompanhar as tendências de mercado e as franquias são classificadas de acordo com seu nível de integração, o que possibilita uma aproximação e propício ao relacionamento (PLÁ, 2001). As mudanças no setor e o amadurecimento do setor levaram as redes consolidadas a levarem em conta a expertise desenvolvida pelos franqueados, criando condições para os conselhos de franqueados participem das decisões (MIUNDZIEV et al., 2011). 


\section{METODOLOGIA}

A análise bibliométrica serve de orientação para os pesquisadores e constitui em objeto de estudo do presente artigo por sua abordagem em pesquisas desenvolvidas. É uma ferramenta que auxilia a organização, identificação e qualificação das informações contidas em publicações acadêmicas, trata-se de um método quantitativo para o estudo do progresso cientifico e tecnológico (VANTI, 2002).

O uso de técnicas bibliométrica contribui de forma decisiva para definir as listas de publicações periódicas e possibilitar a análise das tendências e demandas futuras. Segundo Raan (1996), a análise bibliométrica deve acompanhar a evolução cientifica das publicações dos diversos pesquisadores sobre o mesmo tema. Os métodos bibliométricos são quantitativos na essência, mas podem ser utilizados qualitativamente (WALLIN, 2005).

A análise bibliométrica, nesta pesquisa, delimita o campo de pesquisa na base de dados do Web of Science facilitando o acesso e a organização dos artigos coletados e criando a oportunidade de ampliação dos estudos ligados ao tema, utilizando indicadores que possibilitem a comparação entre as informações obtidas e permite estimar a ocorrência de palavras dos textos científicos pesquisados, a área de concentração, palavras-chaves de um determinado texto científico, a ocorrência das palavras permite a indexação temática automática, análise de citações que permite agrupar as referências bibliográficas incluídas em publicações pelas quais autores e artigos exercem maior influência ou relevância (PRICE, 1965; ARAÚJO, 2006)

O estudo foi realizado a partir do acesso ao Web of Science (2016), a pesquisa foi realizada no dia 02 de setembro de 2016 seguindo o seguinte percurso, respectivamente: foram realizadas busca com a palavra "franchising", sem a delimitação de data, delimitando apenas o campo de pesquisa para Business, Economics, Management e Business Finance. Dessa forma, foram levantadas informações sobre, número total de publicações, áreas temáticas, tipos de publicações, autores, títulos e fontes, instituições, anos de publicações, idiomas e países. A busca originou a seleção de 1101 publicações referente ao tema pesquisado; dos 1101 achados foram selecionadas publicações mais relevantes sobre o tema franchising nas principais análises.

Por conseguinte, ocorreu a elaboração de gráficos e figuras e nuvens de palavras que representassem visualmente a evolução da produção acadêmica, conforme se vê no gráfico 1; a evolução de citação dos quinze artigos mais citados, como se pode visualizar no gráfico 2; as relações dos periódicos com número superior a dez artigos, descritas na tabela 1; a lista de países que mais publicaram artigos sobre o tema, predisposta na tabela 2; as relações das universidades que mais publicam, conforme se nota na tabela 3; a relação das áreas relacionadas ao tema (tabela 4); as nuvens de palavras com palavras chaves dos autores, como se vê na figura 1; e as nuvens de palavras dos abstracts (figura 2).

Por fim, houve a conclusão das análises, utilizando a pesquisa suplementar sobre os autores, por meio de levantamento bibliográfico, bem como leituras total e parcial dos principais artigos. Apesar de a pesquisa utilizar somente dados secundários, o estudo não perde a credibilidade desde que se utilizem de fontes confiáveis que garanta a qualidade e o resultado da pesquisa. Neste trabalho não houve a necessidade 
de realizar a leitura e de todos os artigos, as palavras chaves, as citações, os abstracts, títulos, períodos e autores que foram transformados em gráficos possibilitou a complementação da análise.

\section{RESULTADOS E DISCUSSÃO}

Por meio desta análise bibliométrica é possível constatar que ainda existe um forte interesse acadêmico sobre os temas referentes a franchising. Este fato pode ser observado por meio da crescente produção acadêmica de artigos, intensificada na última década, conforme proposição no gráfico 1, após um declínio na virada do milênio. Alguns fatores exógenos, como o boom da internet, crescimento dos mercados emergentes e impulso no processo de internacionalização das franquias e novos formatos adaptados a esses novos mercados, são aspectos que comprovam a relevância acadêmica do tema.

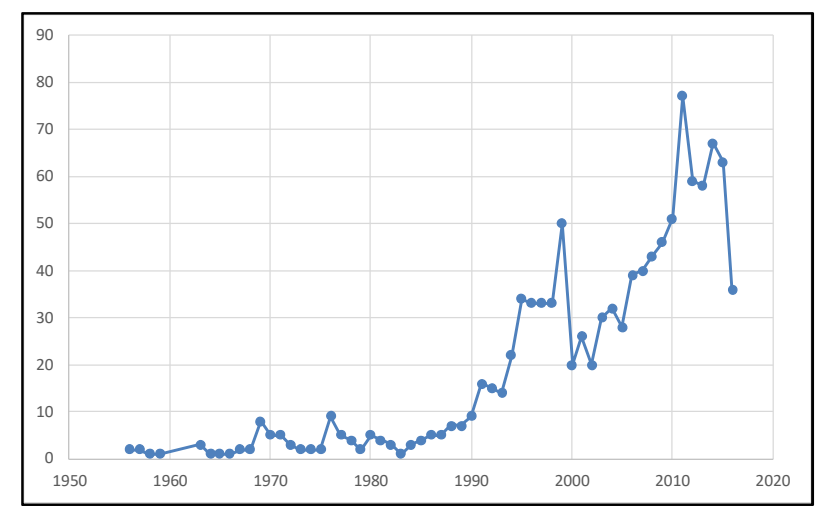

Gráfico 1: Evolução da produção acadêmica em franchising (artigos/ano).

Entre as publicações acadêmicas (gráfico 1) compreendidas no período da pesquisa (1956 a 2016), há um período de crescimento lento que se estende até 1990 (média anual de 3,62 publicações). A partir de 1991 há um crescimento significativo até 1994 (média anual de 16,75 publicações). Entre 1995 (34) e 1999 (50), chega-se a uma média anual de 36,6 publicações, e a seguir, uma forte queda cuja recuperação ocorreu somente uma década depois, em 2010, mas mantendo uma média de 34,1 publicações. No período mais recente 2011 a 2015 passamos a uma média anual de 64,8 publicações. 0 ano de 2016 encontra-se incompleta razão, pela qual foi ignorado.

No final da década de 50, um dos destaques foi a publicação do artigo Automobile Dealer Franchises: Vertical Integration by Contract (KESSLER, 1957). Por tratar de questões contratuais e o pioneirismo no tema, Kessler era pertencente aos quadros da escola de direito de Yale. A publicação trata o surgimento dos dealers, pontos de vendas autorizados limitando-se a revender os produtos da marca licenciada. A escolha do distribuidor e o desenvolvimento do canal por parte dos fabricantes possibilitavam a ampliação do mercado e manutenção do produto, em compartida, espera-se receber do franqueador auxílio no desenvolvimento de ações que contribua positivamente ao incremento do varejo.

Kessler (1957) dá ênfase no setor automobilístico, mas, no entanto, outros segmentos passaram a utilizar o sistema de distribuição. O interesse acadêmico pelo estudo é decorrente da adoção do sistema por várias empresas americanas e também o número crescente de dealers, distribuidores, insatisfeitos além de questões antitrust. A explosão de publicação na década de 90 é decorrente da expansão das redes de 
franquias. Neste período, o esforço principal das redes norte-americanas era buscar o crescimento por meio do desenvolvimento de novos mercados por meio da internacionalização (ROCHA et al., 2014).

Analisando os dados obtidos no Web of Science, os três autores que mais publicação sobre o tema são: Rajiv P. Dant (23 publicações) seguido por Francine Lafontaine (17 publicações) e Patrick Kaufmann (16 publicações). Na sequência temos os trabalhos de Combs, Altinay, Perrigot, Aidt, Michael, Windsperger, Castrogiovanni, Grunhagen, Vazquez, Brookes, Ketchen, Nygaard, Anonymous, Brown, Coates, Frazer, Humphreys, Hunt, Kalnins, Shane, Weaven.

O autor com maior número de publicações é Rajiv P. Dant, sendo que o mesmo é membro da American Marketing Association, Academy of Marketing Science, International Society of Franchising (ISoF) e Society for Marketing Advances. Faz parte do conselho editorial de cinco journals sendo também colaborador em outras seis: Journal of Marketing, Strategic Management Journal, Journal of Retailing, Journal of the Academy of Marketing Science, Journal of Public Policy \& Marketing, Journal of the operational research society, Journal of Business Venturing, Research in Marketing, Marketing Letters, Journal of Business Research, Journal of Small Business Management e Journal of Economic Psychology.

O professor Kaufmann, além de ter sido presidente do International Society of Franchising, no período de 1993-2010, permanecendo como membro até 2014, também possui publicações em uma grande variedade de revistas acadêmicas, incluindo o Journal of Consumer Research, Journal of Marketing, Journal of Law and Economics, Journal of Public Policy \& Marketing, e no Journal of Retailing. Ele atua de modo idêntico no conselho editorial do Journal of Retailing e do Journal of Public Policy \& Marketing.

Nota-se a importância da ISoF que é uma sociedade multidisciplinar internacional para acadêmicos e práticos do franchising que compartilham interesses comuns na promoção das melhores práticas de franchising por meio da educação e da pesquisa. É uma organização formada em 1986 e tem acumulado informações sobre franchising, o crescimento global das operações de franquias, assim como gestão, marketing, relacionamento franqueador/franqueado, empreendedorismo, questões jurídicas e contábeis. Discutem-se também aspectos metodológicos para pesquisas na área.

No período de 1991 a 1994, os temas comuns nas publicações destes autores eram o gerenciamento de conflito, estratégias de distribuição, atividades de marketing, configuração da loja ideal para os franqueados, padronização para fortalecimento da marca. A maturidade do sistema exigia novos formatos com o avanço da tecnologia e da internacionalização, formatos estes que possibilitassem o acesso a mercados emergentes.

Com o amadurecimento do sistema e o surgimento de novas franquias houve interesse maior por parte dos pesquisadores no estudo de políticas públicas, legislação com intuito de evitar comportamentos oportunistas. Neste período a ênfase é a teoria de agência (ALCHIAN et al., 1972; EISENHARDT, 1985, 1989; JENSEN et al., 1976) que trata a relação de contrato como um acordo formal entre as partes, envolvendo questões financeiras envolvendo taxas e pagamento de royalties, questões de propriedade. Os contratos como forma de estabelecer o direto de propriedade dando ênfase nas formas de controle por parte da franqueadora de modo a permitir o alcance de seus objetivos. 
A teoria de agência utilizada para discutir propriedades, compartilhamento de riscos, padronização, regulamentação, cobranças de taxas e tendo como objetivo a discussão da relação franqueador franqueado por meio de contrato. Mais para o final da década com as grandes redes internacionalizadas, o tema é discutido como um método de expansão das redes por meio de compartilhamento da propriedade. Entram na discussão das multiunidades franqueadas, o desenvolvimento da franquia como acesso ao capital, a expansão de novos franqueados, mas também o desenvolvimento de franqueados para que estes possam adquirir outras unidades de franquias.

O estudo avança sobre a taxa e os royalties que são cobrados em busca de uma cooperação vertical, os recursos são utilizados em prol da rede, entendendo que um formato de franchising ou um modelo de cooperação podem produzir retorno e resultados satisfatórios. Neste período Verbierem et al. (2008) escreve sobre a importância do controle no que tange a troca de informações e no desempenho da franquia. A relação do franqueado com o franqueador depende, na maioria das vezes, do desempenho obtido pelo franqueado, os conflitos surgem, normalmente, quando há queda de desempenho.

O estudo não deve mais ficar centrado apenas em franqueadores, mas também se deve considerar o perfil dos franqueados. O conhecimento e a experiência podem oferecer know-how que possibilite aos franqueados gerenciarem áreas de atuação mais amplas permitindo, a propriedade novamente é discutida, mas, desta vez, o foco é no favorecimento de uma maior autonomia para franqueados que possuam multiunidades. Os estudos permeiam mudanças nos formatos o que envolve padronização, o estudo do perfil do empreendedor a fim de identificar potenciais franqueados que possam possibilitar expansão globalizada das redes e melhorar a economia de escala.

Um dos assuntos abordados é o oportunismo por parte dos franqueadores, os estudos apontam que o franqueado não é um mero distribuidor, seus erros e acertos influenciam diretamente a marca, os estudos caminham para evidências do compartilhamento de riscos, o comportamento do franqueado influencia diretamente na reputação da marca. A forte queda, observada no ano 2000 , pode estar relacionada ao crescente volume de mortalidade das pequenas empresas fenômeno este global. No Brasil, a cada 100 organizações que iniciavam as atividades em 2003 apenas 42 empresas sobreviveram até 2007 e das empresas abertas neste período 27 empresas não completaram um ano de vida após a abertura. (ALBUQUERQUE et al., 2011).

A franquia é uma empresa independente, portanto, é necessário que o candidato a franqueado realize a abertura de uma empresa para que o direito seja concedido e possa formalizar o acordo contratual entre ambas por um determinado período de tempo (LAFONTAINE, 1992). Sendo a unidade franqueada uma microempresa as publicações no final da década demonstram uma preocupação com o índice de mortalidade das empresas, um exemplo é o artigo Franchising growth and franchisor entry and exit in the US market: Myth and realy (LAFONTAINE et al., 1998). O tema da mortalidade foi discutido no que tange a sobrevivência das franquias, abordando que a possibilidade de insucesso dependendo de fatores como a idade e a maturidade da franquia. A pesquisa identificou que as novas redes tendem a ter uma taxa de sobrevivência menor e que a escolha do empreendedor pelo sistema de franquias não garantem o sucesso. 
As falhas das unidades franqueadas levaram os autores (LAFONTANE et al., 1999; KAUFAMANN, 1999) a ampliar a pesquisa, passando a entender os conflitos e para entendê-los traçar perfis dos franqueados, entendo o franchising como um modelo de cooperação pelas quais a troca de informações são fatores importantes. As pesquisas no final da década buscam entender a realidade no que tange a busca de desempenho a partir dos estudos do perfil do franqueado e o compartilhamento de risco, entendendo a tendência ao oportunismo de ambas as partes, por um lado, o franqueador na busca de capital e por outro lado, o franqueado responsabilizando a franqueadora pelo seu fracasso na busca pelo chamado auto emprego (KAUFMANN, 1999).

O boom em publicação em 1999 e a brusca queda nos anos subsequentes na produção acadêmica evidenciam, provavelmente, a tentativa de explicar, no primeiro momento, os fracassos experimentados pelo sistema. Em um segundo momento, a dificuldade de encontrar indícios além do impacto do atentado de 11 de setembro de 2001 na sociedade americana de um modo geral e nas pesquisas realizadas naquele país em particular.

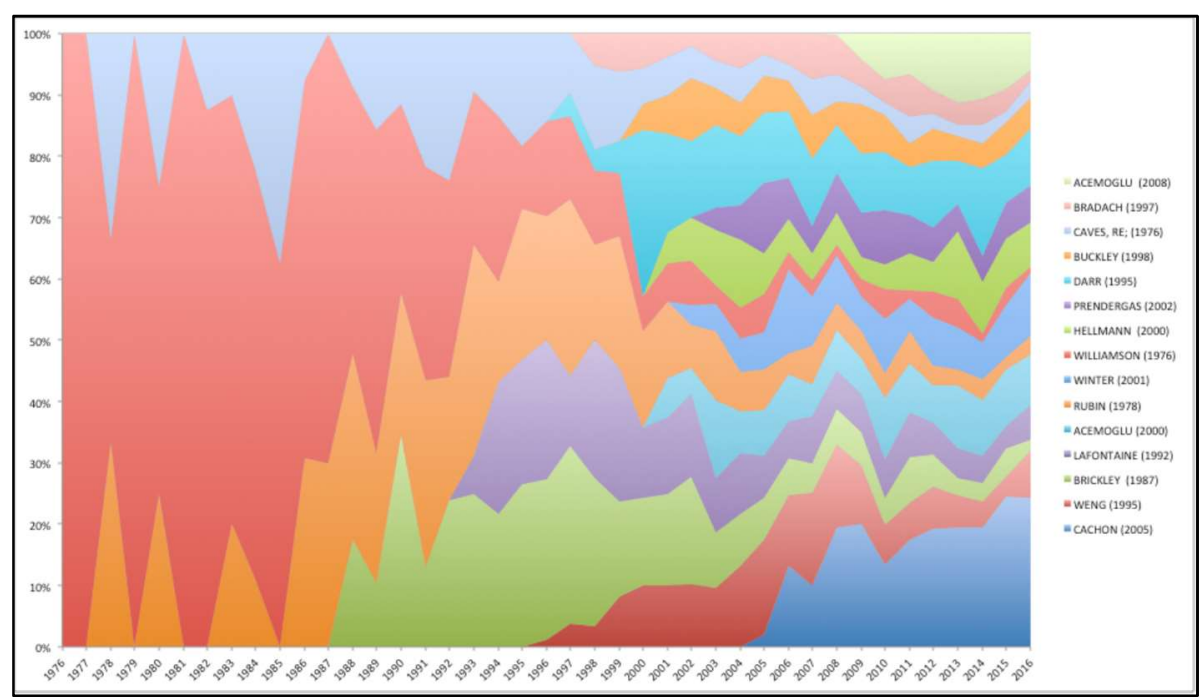

Gráfico 2: Evolução das citações dos artigos "Top 15".

Os trabalhos com maior número de citação são apresentados pelo gráfico 2. Devemos observar que a leitura do mesmo é feito na vertical, por ano, sendo a somatória das áreas igual a $100 \%$. Exemplo é o trabalho de Williamson (1976) com 100\% em 1976 e 1977. Em ordem decrescente, do maior número de citações para o menor tem-se: Cachon et al. (2005); o mais citado e em crescimento, Weng (1995); ainda citado, mas perdendo força, Brickley et al. (1987), assim como Lafontaine (1992) foram trabalhos muito citados na década de noventa, mas com contribuição marginal atualmente. Acemoglu et al. (2000), com citações recentes, mas estabilizadas.

Rubin (1978) mantém citações ao longo do tempo, mas com pouca contribuição atual, Winter et al. (2001) continuam com citações referentes a questão dos fluxos de conhecimento, como a existente na relação franqueador-franqueado. Williamson (1976) já foi o mais citado até início da década de 90, mas atualmente, com uma contribuição marginal. Hellmann et al. (2000), Prendergast (2002), Buckley et al. (1998) estão mantendo sua média de citações. Darr et al. (1995) já teve maior número de citações mas mantém um bom número destas. Caves et al. (1976), por ser pioneiro, hoje mantém um menor número de citações do 
que no passado; Bradach (1997), hoje com citações residuais; e, por fim, Acemoglu et al. (2008) com citações recentes, mas não sustentáveis. É pertinente também para uma análise bibliométrica destacar os principais periódicos que contribuem para os estudos sobre o tema franchising, conforme se vê na tabela 1.

Tabela 1: Relação dos periódicos com mais de 10 artigos.

\begin{tabular}{|l|r|r|}
\hline Journal & \# & de 1101 \\
\hline Journal of retailing & 41 & $3,7 \%$ \\
\hline Service industries journal & 39 & $3,5 \%$ \\
\hline Journal of small business management & 35 & $3,2 \%$ \\
\hline Journal of business venturing & 33 & $3,0 \%$ \\
\hline Journal of business research & 25 & $2,3 \%$ \\
\hline Forbes & 24 & $2,2 \%$ \\
\hline Journal of sport management & 19 & $1,7 \%$ \\
\hline European journal of marketing & 17 & $1,5 \%$ \\
\hline Review of industrial organization & 16 & $1,5 \%$ \\
\hline Applied economics & 15 & $1,4 \%$ \\
\hline Management science & 14 & \\
\hline Journal of institutional and theoretical economics zeitschrift fur & 14 & $1,3 \%$ \\
\hline Journal of marketing & & \\
\hline Rand journal of economics & 13 & $1,2 \%$ \\
\hline European journal of operational research & & \\
\hline Journal of law economics & & \\
\hline Journal of regulatory economics & & \\
\hline Strategic management jornal & 12 & $1,1 \%$ \\
\hline Cornell hospitality quarterly & & \\
\hline Entrepreneurship theory and practice & & \\
\hline Southern economic jornal & 11 & \\
\hline International journal of contemporary hospitality management & & \\
\hline Journal of economic behavior organization & & \\
\hline Journal of taxation & & \\
\hline Journal of law economics organization & \\
\hline Journal of transport economics and policy & \\
\hline Public choice & \\
\hline \hline
\end{tabular}

As principais fontes de publicação no tema são: Journal of Retailing, Service Industries Journal, Journal of Small Business Management, Journal of Business Venturing, Journal of Business Research. Desse modo há evidencias que os veículos de publicação são diversificados. Além disso, esses são alguns dos periódicos preferidos dos autores mais prolíficos. A revista de negócios Forbes é conhecida por apresentar rankings de organizações mais valiosas. No caso das franquias são conhecidos os rankings das franquias esportivas mais valiosas para diversas modalidades incluindo as poderosas NBA (National Basketball Association) e NFL (National Football League) norte-americanas.

Periódicos específicos como o Journal of Sport Management apresentam uma área de forte crescimento no tema, assim como outros tem uma forte ligação com o marketing como o European Journal of Marketing, Journal of Marketing. Áreas ligadas à economia e suas conexões incluindo aspectos políticos são representados por publicações como: Review of Industrial Organization, Applied Economics, Journal of Institutional and Theoretical Economics, Rand Journal of Economics, Journal of Regulatory Economics, Southern Economic Journal, Journal of Economic Behavior Organization, Journal of Transport Economics and Policy e Public Choice.

Journals de alto impacto entre os mais tradicionais encontram-se o Management Science e o Strategic Management Journal. Para área de pesquisa operacional tem-se o European Journal of Operational Research. Áreas como legislação e impostos são representadas por periódicos como o Journal of Law Economics, Journal of Taxation e o Journal of Law Economics Organization. Para a área de hospitalidade tem- 
se Cornell Hospitality Quarterly, International Journal of Contemporary Hospitality Management e no empreendedorismo a Entrepreneurship Theory and Practice.

Línguas de publicação tem-se o inglês como majoritário com 1066 artigos, seguidos pelo espanhol (dez), francês (seis), português (cinco), alemão, russo e eslovaco com quatro e, por fim, com duas publicações no idioma tcheco. Das cinco publicações apontadas como publicadas no idioma português, uma delas Monge e Huerta-Zavala (2015), na verdade foi publicado em língua espanhola, as demais quatro publicações efetivamente em língua portuguesa, Lacerda et al. (2015), Lopes et al. (2010), Peçanha et al. (2015) e Vaz et al. (2015). Todos foram publicados em periódicos brasileiros: Revista Evidenciação Contábil \& Finanças, Revista Brasileira de Gestão de Negócios, Revista de Administração de Empresas, Revista de Gestão e Tecnologia e Revista Contabilidade e Controladoria.

Tabela 2: Lista dos países que mais publicam artigos sobre o tema.

\begin{tabular}{|l|r|r|}
\hline País & \# & \% de $\mathbf{1 1 0 1}$ \\
\hline USA & 606 & $55,0 \%$ \\
\hline ENGLAND & 114 & $10,4 \%$ \\
\hline SPAIN & 69 & $6,3 \%$ \\
\hline CANADA & 54 & $4,9 \%$ \\
\hline AUSTRALIA & 44 & $4,0 \%$ \\
\hline FRANCE & 43 & $3,9 \%$ \\
\hline GERMANY & 35 & $3,2 \%$ \\
\hline PEOPLES R C & 25 & $2,3 \%$ \\
\hline ITALY & 20 & $1,8 \%$ \\
\hline TAIWAN & 19 & $1,7 \%$ \\
\hline NORWAY & 15 & $1,4 \%$ \\
\hline SCOTLAND & & \\
\hline AUSTRIA & 14 & $1,3 \%$ \\
\hline NETHERLANDS & 12 & $1,1 \%$ \\
\hline SOUTH KOR & 10 & $0,9 \%$ \\
\hline BRAZIL & & \\
\hline CHILE & & $0,8 \%$ \\
\hline ISRAEL & & \\
\hline JAPAN & & \\
\hline SWITZERLAND & & \\
\hline SINGAPORE & & \\
\hline DENMARK & & \\
\hline NEW ZEALAND & & \\
\hline NORTH IRELAND & & \\
\hline TURKEY & & \\
\hline
\end{tabular}

Um aspecto relevante para a pesquisa em franchising é a sua alta concentração em países de língua inglesa (EUA, Inglaterra, Canadá e Austrália). Poucos países em desenvolvimento iniciam um processo de publicação no tema como a China (25 papers), Taiwan (19 papers), Coréia do Sul (12 papers), Brasil e Chile com dez papers e Turquia com sete publicações. Dessa forma o tema ainda é predominante em países de economia madura e a maioria deles pertencentes à OCDE (Organização para a Cooperação e Desenvolvimento Econômico).

Em relação às instituições uma alta concentração em Universidades e não em organismos internacionais como Banco Mundial. Dessa forma observamos que as pesquisas estão concentradas em centros de pesquisa de universidades norte-americanas como as Universidades de Michigan, Boston e Illinois no nordeste norte-americano, Em seguida Universidades Australianas como a Griffith University que é um centro de excelência em franchising e britânicas (Cambridge). Vale a observação da mobilidade de pesquisadores como, por exemplo, de Boston para Oklahoma. Outro aspecto é o da coautoria entre Universidades americanas e européias. 
Tabela 3: Relação das Universidades que mais publicam.

\begin{tabular}{|l|r|r|}
\hline Universidades & \% de 1101 \\
\hline UNIV MICHIGAN & 25 & $2,3 \%$ \\
\hline BOSTON UNIV & 20 & $1,8 \%$ \\
\hline UNIV ILLINOIS & 20 & $1,8 \%$ \\
\hline GRIFFITH UNIV & 18 & $1,6 \%$ \\
\hline UNIV CAMBRIDGE & 16 & $1,5 \%$ \\
\hline UNIV FLORIDA & 15 & $1,4 \%$ \\
\hline UNIV OKLAHOMA & 15 & $1,4 \%$ \\
\hline CORNELL UNIV & 14 & $1,3 \%$ \\
\hline OXFORD BROOKES UNIV & 14 & $1,3 \%$ \\
\hline TEXAS A M UNIV & 14 & $1,3 \%$ \\
\hline UNIV ALBERTA & 14 & $1,3 \%$ \\
\hline UNIV RENNES 1 & 14 & $1,3 \%$ \\
\hline UNIV VIENNA & 13 & $1,2 \%$ \\
\hline AUBURN UNIV & 12 & $1,1 \%$ \\
\hline GEORGIA STATE UNIV & 12 & $1,1 \%$ \\
\hline PENN STATE UNIV & 12 & $1,1 \%$ \\
\hline UNIV N CAROLINA & 12 & $1,1 \%$ \\
\hline UNIV SO CALIF & 12 & $1,1 \%$ \\
\hline UNIV TEXAS & 12 & $1,1 \%$ \\
\hline FLORIDA STATE UNIV & 11 & $1,0 \%$ \\
\hline MIT & 11 & $1,0 \%$ \\
\hline STANFORD UNIV & 11 & $1,0 \%$ \\
\hline UNIV PENN & 11 & $1,0 \%$ \\
\hline
\end{tabular}

Um fator que deve ser destacado é a questão da mobilidade de pesquisadores que ao desenvolverem pesquisas em uma Universidade acabam transferindo essas pesquisas para outras Universidades, muitas vezes, em continentes distintos. Outras parcerias ocorrem, por exemplo, entre centros de pesquisas de Universidades norte-americanas e européias. O autor Radij P. Dant, é doutor pela Universidade da Virgínia onde fez carreira, passou por várias outras Universidades, destacando-se: Boston University, University Oklahoma, University North Carolina e Florida State University. Dentre as Universidades que mais publicam sobre o tema, destaca-se a University Michigan que tem como professora e pesquisadora, Francine Lafontaine. A Universidade de Boston é a segunda no ranking e tem como principal autor e pesquisador, vinculado ao tema, o professor Patrick Kaufmann.

$\mathrm{Na}$ análise notam-se também Universidades fora dos Estados Unidos, como a Universidade de Rennes na França que tem como pesquisadores vinculados ao programa os autores Rozenn Perrigot e Gerard Cliquet, que possuem publicações em coautoria com Radij P. Dant. Uma observação importante é de que o tema franchising tem diversas conexões com outras áreas (Tabela 4) que não a gestão ou a economia. Um destaque é a sua relação com ciências sociais e em especial com o direito por se tratar de configurações organizacionais dependentes de relações contratuais. A sua relação com a logística (operações) e transporte também é destacada e tem relação com a expansão territorial típica do modelo. São tratados também pela psicologia e sociologia devidos as relações conflituosas e aspectos culturais que leva ao estudo do comportamento das organizações.

Algumas áreas que tem alta correlação são também identificadas e correspondem a atividades ligadas a ciência dos esportes. Nota-se que é tradicional nos EUA o uso do termo franquias esportivas como as encontradas na NBA. Uma lacuna encontrada é relacionada com a área de serviços e mesmo de trabalhos na área de pesquisa educacional. 
Tabela 4: Relação das áreas relacionadas ao tema.

\begin{tabular}{|l|r|r||}
\hline Áreas de Pesquisa & $\#$ & \% de 1101 \\
\hline Business economics & 1101 & $100,0 \%$ \\
\hline Social sciences other topics & 84 & $7,6 \%$ \\
\hline Government law & 61 & $5,5 \%$ \\
\hline Operations research management science & 49 & $4,5 \%$ \\
\hline Transportation & 24 & $2,2 \%$ \\
\hline Psychology & 20 & $1,8 \%$ \\
\hline Sociology & 19 & $1,7 \%$ \\
\hline Sport sciences & & \\
\hline Public administration & 18 & $1,6 \%$ \\
\hline Environmental sciences ecology & 12 & $1,1 \%$ \\
\hline Urban studies & & \\
\hline Communication & 8 & $0,7 \%$ \\
\hline Mathematical methods in social sciences & & \\
\hline Mathematics & & \\
\hline Engineering & 6 & $0,5 \%$ \\
\hline Information science library science & 5 & $0,5 \%$ \\
\hline Agriculture & 4 & $0,4 \%$ \\
\hline Computer science & & \\
\hline History & & \\
\hline Energy fuels & 3 & $0,3 \%$ \\
\hline Geography & & \\
\hline International relations & & \\
\hline Area studies & 2 & $0,2 \%$ \\
\hline Ethnic studies & & \\
\hline Telecommunications & 2 & $0,2 \%$ \\
\hline Education educational research & 1 & $0,1 \%$ \\
\hline \hline
\end{tabular}

As palavras-chaves, propostas na figura 1, utilizadas com uma frequência maior nas publicações do tema franchising foram: firm, form, agency, performance, theory, ownwership, contracts, management, systems, integration, choice, francise, growth, behavior, informations, relationships. Pode-se notar que uma análise inicial nos remete ao próprio desenvolvimento da pesquisa na área, proposta na figura 1 onde trabalhos iniciais de Williamson (1976) e Caves et al. (1976) nos remetem a contratos, relacionamentos e a teoria da agência. A questão central da estratégia também é destacada como a presença do termo performance. Outras mais associadas a estrutura como forma, e a questão da governança como comportamento e as escolhas. Outras associadas com a cadeia como integração, sistemas, informação, canais.

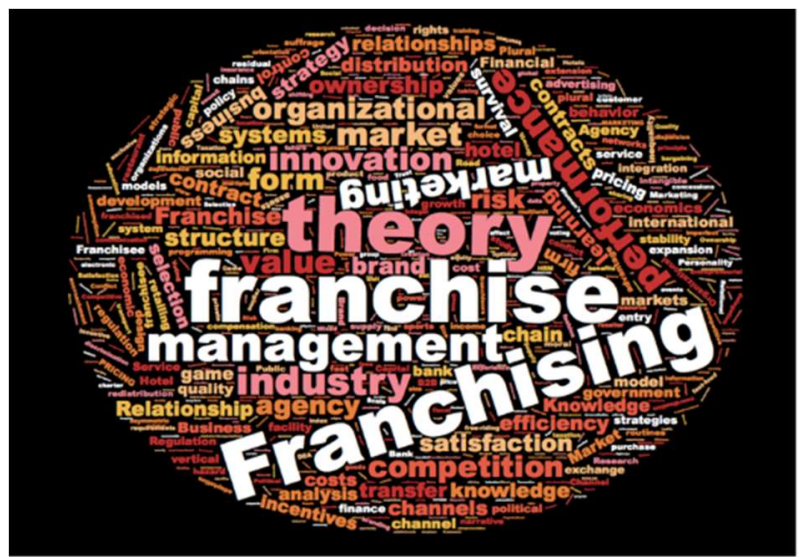

Figura 1: Nuvem de palavras - palavras-chave (autores).

Em relação a nuvem de palavras que demonstra a frequência encontrada nos abstracts (Figura 2) é constante os diversos termos relacionados ao assunto tema da pesquisa: franchise(d), franchising, franchisees, franchisor(s), franchisee e franchises. As palavras a seguir constituem uma grande frequência: 
model, Market, firms, international, rights, system(s), relationship(s), theory, brand, contract(s), performance, value, service(s), units, knowledge, ownership(s), risk, management, support, form, channel, control, chains, competition, industry, cost(s), quality, strategy, agency, economic, capital, outlets, growth, advertising, development, structure.

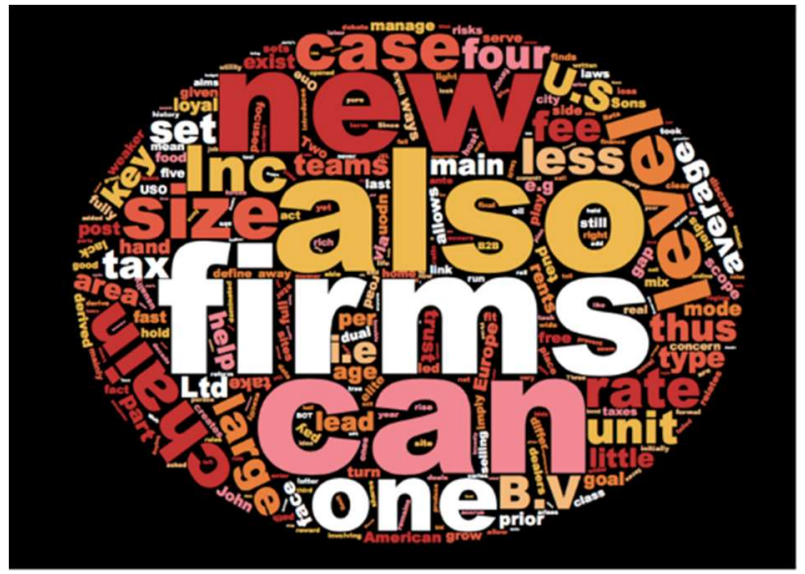

Figura 2: Nuvem de palavras - abstract.

Uma visão dos abstracts amplia consideravelmente a visão do próprio conceito de franchising com preocupações que vão desde o capital e as fontes de financiamento rumo a um crescimento sustentável e sua performance econômica. É destacada também os aspectos ligados diretamente à estrutura tais como: unidades, canais, outlets, cadeias e a sua internacionalização, assim como destacado aspectos de marketing como marca, custos e propaganda.

\section{CONSIDERAÇÕES FINAIS}

Este artigo conduziu uma revisão do estado da arte sobre pesquisa em franchising. Os principais autores em relação a número de publicações e journals de todo o período da pesquisa (1956-2016) nos apresentam a evolução da disciplina. Os dados foram extraídos unicamente da Web of Science que foram utilizados não somente para os papers publicados, mas diversas informações com destaque para as citações.

$\mathrm{O}$ assunto tem crescido em importância e destaque em alguns períodos de maior produtividade que corresponde a marcos como uma maior internacionalização. Uma análise interessante é que a pesquisa no tema é bastante diversificada passando por áreas como o empreendedorismo, a economia e o direito, em especial, pelas questões contratuais e de conflitos.

A análise das citações possibilitou identificar os autores mais importantes numa linha do tempo. Disso tem-se uma mudança conceitual que vem de autores clássicos como Williamson (1976) que fez uma análise do direto da propriedade, avaliando a natureza dos contratos de franquias. Na época o sistema de franchising despertou interesse da academia, em especial dos estudos econômicos por se tratar de uma relação econômica entre os agentes. Para consolidar a hipótese, autores revisitaram a teoria da agência para analisar o fenômeno do sistema de franchising que compreende nas relações contratuais entre as firmas, distintas das relações contratuais existentes em outras empresas fora do setor. Outros autores como Rubin (1978), Caves e Murphy (1976) foram também motivados a pesquisar sobre o tema, visto na época como 
alternativa de obtenção de capital para o negócio. A abordagem da época encontrou respaldo na teoria da agência.

No período seguinte, na década de 80, o destaque para Brickley et al. (1987), que ampliaram a pesquisa sobre o direito de propriedade. Os problemas de agência ou de direito à propriedade podem ser maiores se não forem previsto no contrato. Dessa forma, o direito de propriedade pode variar entre a concessão por uma unidade franqueada e a autorização de uma determinada unidade franqueada assumir uma localidade, tornando-se subsidiária da franqueadora.

Nos anos 90, período de maior índice de publicações sobre o tema, acredita que o direcionamento passou por dois momentos importantes, o primeiro de (1991-1994) e o segundo momento de (1995-1999). No primeiro momento, a abordagem era sobre o amadurecimento do sistema, o surgimento de novas redes, aumento da competividade, a preocupação com desempenho e os conflitos, bem como, os comportamentos oportunistas. As pesquisas voltavam para melhoria da gestão por meio das estratégias de ampliação de mercado e atividades de marketing. Neste período os autores que mais publicaram conforme o levantamento e análise realizada por meio do Web of Science foram Dant (23 publicações), Lafontaine (17 publicações) e Kaufmann (16 publicações). Destaque para Lafontaine que também está na lista dos mais citados.

No segundo momento, (1995-1999), o destaque em publicações ordenadas pelo número de citações é: Weng (1995), Buckley et al. (1998), e Darr et al. (1995). As abordagens dos pesquisadores estão relacionadas ao estudo do perfil do empreendedor, transferência de conhecimento, know-how, autonomia, expansão globalizada das redes, economia de escala. O estudo neste período caminha para o compartilhamento de risco. Apesar de autor Kaufmann (KAUFMANN, 1999; KAUFMANN et al., 1996; KAUFMANN et al., 1999; KAUFMANN et al., 1994; KAUFMANN et al., 1990; KAUFMANN et al., 1995) não estar entre os mais citados, ele aparece com publicações no período.

Após o ápice de publicações em 1999, houve uma queda significativa nas quedas das publicações, recuperando somente em 2010. As obras publicadas neste período, ordenadas de acordo com as citações foram os artigos autores Hellmann et al. (2000) e Prendergast (2002). Hellmann et al. (2000) e Prendergast (2002) foram descartados por não tratar diretamente sobre o tema, abordagem envolve finanças, risco, problemas de agência.

Destaca-se neste período, a pesquisa sobre a gestão da marca no segmento de franchising, taxa de propaganda, royalties, focando na gestão empresarial, assuntos abordados nos trabalhos dos autores: Lafontaine et al. (2005) e Dant et al. (2003). Nesta década, vale destacar autores como Cachon et al. (2005), o mais citado em nos dias atuais ainda em crescimento em citações, o que demostra um interesse da academia em estudar o sistema de franchising como um formato de varejo, aspectos relacionados à cadeia de suprimentos, preço de venda, ações no ponto de venda e aspectos gerais na estrutura da loja.

Avaliando as publicações, percebem-se fontes diversificadas; no entanto, houve uma migração, um direcionamento no interesse dos pesquisadores, ao longo do tempo, reduziram o número de publicações em periódicos da área econômica e uma migração para outros periódicos, principalmente, os periódicos relacionados a negócios, especialmente os ligados à área de marketing. 
Os indícios para pesquisas futuras indicam o estudo sobre o franchising na perspectiva varejista, estudo do e-commerce, redução de custos nas transações. O tema tecnologia é bastante discutido como formas de comercialização de produto ou serviço, bem como, o seu uso no gerenciamento. Destaca-se também, a aplicação da visão baseada em recursos conforme artigos mais recentes como: Determinants of E-Commerce Strategy in Franchising: A Resource-Based View e Franchisor-franchisee supply chain cooperation: Sharing of demand forecast information in high-tech industries. Os artigos confirmam a convergência dos estudos de universidades europeias e americanas para formatos que utilizem a tecnologia. Outra tendência aponta para estudos interorganizacionais como alternativa de entender as relações entre franqueador-franqueado, a qualidade do relacionamento, a maturidade da rede, dimensões da personalidade.

O volume de publicações concentra-se em países de língua inglesa (USA, Inglaterra, Canadá, Austrália), um exemplo, é o artigo Influence of personality traits on perceived relationship quality within a franchisee franchisor em parceria com pesquisadores americanos e australianos. Dentre os centros de pesquisas fora dos Estados Unidos, quem mais possui publicações relevantes sem a coautoria americana é a Universidade de Rennes, na França.

Verificou-se, também, que os países em desenvolvimento possuem baixos índices de publicações. Mas independente da procedência da publicação ou do centro de pesquisa, tanto a análise das palavraschaves com a dos abstracts convergem como estudo de franchising assuntos como: relação contratual, relacionamentos e a teoria da agência, estrutura, questão de governança, cadeia de suprimentos, canais, informação, crescimento sustentável, internacionalização, desempenho, aspectos relacionados a marketing como marca e custo de propaganda.

O escopo deste artigo tem suas limitações como o uso unicamente da web of Science. Novos trabalhos podem utilizar a fusão com bases como a Scopus ou mesmo bases de dados nacionais como o SPELL não integrado ao web of Science diferentemente do Scielo. Mesmo com a ampliação do escopo existem raríssimos trabalhos sobre o tema relacionados a serviços, educação e sobrevivência e mortalidade das redes de franquias.

Acredita-se que este artigo possa servir de ponto de partida para vários outros trabalhos de pesquisa que investigue sobre a sobrevivência das redes de franquias, que avalie como as redes de ensino superior vêm utilizando-se do sistema de franchising para ampliar os seus negócios e, também, estudos relacionados à operação de serviços nas redes de franquias.

O estudo apontou interesses acadêmicos em pesquisar o tema franchising no ponto de vista dos estudos organizacionais, envolvendo aspectos das relações interorganizacionais, tecnologia, varejo, marketing e do ponto vista estratégico, utilizando-se da visão baseadas em recurso, criação de multiformatos é discutida principalmente sob aplicação da tecnologia como parte dos modelos de negócios. $O$ tema é relevante aos pesquisadores brasileiros pelo fato de ser um modelo de negócio em constantes mudanças e desenvolvimento que convergem com os aspectos da situação de países que buscam crescimento. 


\section{REFERÊNCIAS}

ACEMOGLU, D.; ROBINSON, J. A.. Why did the west extend the franchise? Democracy, inequality, and growth in historical perspective. Quarterly Journal of Economics, v.115, n.4, p.1167-1199, 2000.

ALBUQUERQUE, A. F.; ESCRIVÃO FILHO, E.. Fatores de mortalidade de pequenas empresas: uma análise da produção acadêmica no período 2000-2010. ENCONTRO DE ESTUDOS EM ESTRATÉGIA, 3. Anais. Porto Alegre: ANPAD, 2011.

ALBUQUERQUE, L. G.; ANDRADE, M. M. T.. A inovação em uma rede de franchising: estudo do caso Yázigi. Revista de Administração da Universidade de São Paulo, v.31, n.2, 1996.

ALCHIAN, A.; DEMSETZ, H.. Production, information costs, and economic organization. American Economic Review, v.62, n.5, p.777-795, 1972.

ANGELO, C. F. D.; FOUTO, N. M. M. D.; NIELSEN, F. A. G.. Manual do varejo no Brasil. São Paulo: Saint Paul, 2012.

ARAÚJO, C. A.. Bibliometria: evolução histórica e questões atuais. Em Questão, Porto Alegre, v.12, n.1, p.11-32, 2006.

ARAÚJO, R. F.; ALVARENGA, L.. A bibliometria na pesquisa científica da pós-graduação brasileira de 1987 a 2007.

Revista Eletrônica de Biblioteconomia e Ciência da Informação, v.16, n.31, p.51-70, 2011.

BRADACH, J. L.. Using the plural form in the management of restaurant chains. Administrative Science Quarterly, v.42, n.2, p.276-303, 1997.

BRICKLEY, J. A.. Incentive conflicts and contractual restraints: evidence from franchising. Journal of Law \& Economics, v.42, n.2, p.745-774, 1999.

BRICKLEY, J. A.; DARK, F. H.. The choice of organizational form: the case of franchising. Journal of Financial Economics, v.18, n.2, p.401-420, 1987.

BUCKLEY, P. J.; CASSON, M. C.. Analyzing foreign market entry strategies: extending the internalization approach. Journal of International Business Studies, v.29, n.3, p.539561, 1998.

CACHON, G. P.; LARIVIERE, M. A.. Supply chain coordination with revenue-sharing contracts: strengths and limitations. Management Science, v.51, n.1, p.30-44, 2005.

CAVES, R. E.; MURPHY, W. F.. Franchising: firms, markets, and intangible assets. Southern Economic Journal, v.42, n.4, p.572-586, 1976.

\section{CHERTO, M.; CAMPORA, F.; DALL'OLLIO, C.. Mas que}

franchising: como utilizar o que dá certo nas franquias para otimizar o desempenho de outros canais vendas. São Paulo: Premier Máxima, 2006.

COMBS, J. G.; MICHAEL, S. C.; CASTROGIOVANNI, G. J.. Franchising: a review and avenues to greater theoretical diversity. Journal of Management, Thousand Oaks/CA, v.30, n.6, p.907-931, 2004.
DANT, R. P.. A futuristic research agenda for the field of franchising. Journal of Small Business Management, v.46, n.1, p.91-98, 2008.

DANT, R. P.; BERGER, P. D.. Modelling cooperative advertising decisions in franchising. Journal of the Operational Research Society, v.47, n.9, p.1120-1136, 1996.

DANT, R. P.; GUNDLACH, G. T.. The challenge of autonomy and dependence in franchised channels of distribution. Journal of Business Venturing, v.14, n.1, p.35-67, 1999.

DANT, R. P.; KAUFMANN, P. J.. Structural and strategic dynamics in franchising. Journal of Retailing, v.79, n.2, p.6375, 2003.

DANT, R. P.; KAUFMANN, P. J.; PASWAN, A. K.. Ownership redirection in franchised channels. Journal of Public Policy \& Marketing, v.11, n.1, p.33-44, 1992.

DANT, R. P.; NASR, N. I.. Control techniques and upward flow of information in franchising in distant markets: conceptualization and preliminary evidence. Journal of Business Venturing, v.13, n.1, p.3-28, 1998.

DANT, R. P.; PASWAN, A. K.; KAUFMAN, P. J.. What we know about ownership redirection in franchising: $A$ meta-analysis. Journal of Retailing, v.72, n.4, p.429-444, 1996.

DANT, R. P.; PERRIGOT, R.; CLIQUET, G.. A cross-cultural comparison of the plural forms in franchise networks: United States, France, and Brazil. Journal of Small Business Management, v.46, n.2, p.286-311, 2008.

DANT, R. P.; SCHUL, P. L.. Conflict-resolution processes in contractual channels of distribution. Journal of Marketing, v.56, n.1, p.38-54, 1992.

DANT, R. P.; WEAVEN, S. K.; BAKER, B. L.. Influence of personality traits on perceived relationship quality within a franchisee-franchisor context. European Journal of Marketing, v.47, n. 2, p.279-302, 2013.

DARR, E. D.; ARGOTE, L.; EPPLE, D.. The acquisition, transfer, and depreciation of knowledge in service organizations: Productivity in franchises. Management Science, v.41, n.11, p.1750-1762, 1995.

EISENHARDT, K. M.. Control: Organizational and economic approaches. Management Science, v.31, n.2, p.134, 1985.

EISENHARDT, M. K.. Agency theory: An assessment and review. Academy of Management Review, v.14, n.1, p.57, 1989.

ELANGO, B.; FRIED, V. H.. Franchising research: A literature review and synthesis. Journal of Small Business Management, v.35, n.3, p.68-81, 1997.

FRIEDHEIM, A.. Newsletter ABF. São Paulo: ABF, 2013.

HELLMANN, T. F.; MURDOCK, K. C.; STIGLITZ, J. E.. Liberalization, moral hazard in banking, and prudential regulation: Are capital requirements enough?. American Economic Review, v.90, n.1, p.147-165, 2000. 
JENSEN, M.; MECKLING, W.. Theory of the firm: Managerial behavior, agency costs, and ownership structure. Journal of Financial Economics, v.3, p.305-360, 1976.

KAPLAN, R. S.; NORTON, D. P.. Organização orientada para a estratégia: como as empresas que adotam o balanced scorecard prosperam no novo ambiente de negócios. Houston: Gulf Professional Publishing, 2000

KAUFMANN, P. J.. Franchising and the choice of self employment. Journal of Business Venturing, v.14, n.4, p.345-362, 1999.

KAUFMANN, P. J.; DANT, R. P.. Multi-unit franchising: Growth and management issues. Journal of Business Venturing, v.11, n.5, p.343-358, 1996.

KAUFMANN, P. J.; EROGLU, S.. Standardization and adaptation in business format franchising. Journal of Business Venturing, v.14, n.1, p.69, 1999.

KAUFMANN, P. J.; LAFONTAINE, F.. Costs of control: the source of economic rents for McDonald's franchisees. Journal of Law \& Economics, v.37, n.2, p.417-453, 1994.

KAUFMANN, P. J.; RANGAN, V. K.. A model for managing system conflict during franchise expansion. Journal of Retailing, v.66, n.2, p.155-173, 1990.

KAUFMANN, P. J.; STANWORTH, J.. The decision to purchase a franchise: a study of prospective franchisees. Journal of Small Business Management, v.33, n.4, p.22-33, 1995.

KESSLER, F.. Automobile dealer franchises: vertical integration by contract. The Yale Law Journal, v.66, n.8, p.1135-1190, 1957.

LACERDA, R. A. A.; LIMA, N. C.; SOUZA, G. H. S.. Programmed costs versus actual costs: the case of a fast-food franchise. Revista Evidenciação Contábil \& Finanças, v.3, n.1, p.84-99, 2015.

LAFONTAINE, F.. Agency theory and franchising: some empirical results. Rand Journal of Economics, v.23, n.2, p.263-283, 1992.

LAFONTAINE, F.. Contractual arrangements as signaling devices: evidence from franchising. Journal of Law Economics \& Organization, v.9, n.2, p.256-289, 1993.

LAFONTAINE, F.; KAUFMANN, P. J.. The evolution of ownership patterns in franchise systems. Journal of Retailing, v.70, n.2, p.97-113, 1994.

LAFONTAINE, F.; SHAW, K. L.. Franchising growth and franchisor entry and exit in the US market: Myth and reality. Journal of Business Venturing, v.13, n.2, p.95-112, 1998.

LAFONTAINE, F.; SHAW, K. L.. Targeting managerial control: evidence from franchising. Rand Journal of Economics, v. 36, n. 1, p. 131-150, 2005. ISSN 0741-6261.

LAFONTAINE, F.; SHAW, K. L.. The dynamics of franchise contracting: Evidence from panel data. Journal of Political Economy, v.107, n.5, p.1041-1080, 1999.
LAFONTAINE, F.; SLADE, M. E.. Retail contracting: Theory and practice. Journal of Industrial Economics, v.45, n.1, p.1-25, 1997.

LAL, R.. Improving channel coordination through franchising. Marketing Science, v.9, n.4, p.299-318, 1990.

LOPES, H. E. G.; MOURA, T. N.; OLIVEIRA, C. C. G.. Strategic profiles in Brazil: a study of postal agents franchisees from the typology of Miles and Snow. Revista Brasileira De Gestão De Negócios, v.12, n.37, p.388-404, 2010.

MELO, P. L. R.. Internacionalização das redes de franquias brasileiras: uma análise organizacional e institucional. Tese (Doutorado). Universidade de São Paulo, São Paulo, 2012.

MIUNDZIEV, N.; WINDSPERGER, J.. The struture of decision right in franchising networks. Waco: Baylor University, 2011.

MONGE, M. E. C.; HUERTA-ZAVALA, P. A.. Franchisee net income: a signal to choose a franchise in a period of crisis. Revista De Administração De Empresas, v.55, n.6, p.688698, 2015.

PECANHA, A. R. D.; DE OLIVEIRA, S. B.. Family business, yes! But what is the problem, if its management is professional?. Navus - Revista de Gestão e Tecnologia, v.5, n.3, p.126-135, 2015.

PERRIGOT, R.; PÉNARD, T.. Determinants of e-commerce strategy in franchising: a resource-based view. International Journal of Electronic Commerce, v.17, n.3, p.109-130, 2013.

PLÁ, D.. Tudo sobre franchising. Rio de Janeiro: Senac, 2001.

PRADO, M.. Natureza Jurídica do Franchising. São Paulo: Atlas 2012.

PRENDERGAST, C.. The tenuous trade-off between risk and incentives. Journal of Political Economy, v.110, n.5, p.10711102, 2002.

PRICE, D. S.. Networks of Scientific Papers: the pattern of bibliographic references indicates the nature of the scientific research front. Science, v.149, p.510-515, 1965.

RAAN, A. V.. Advanced bibliometric methods as quantitative core of peer review based evaluation and foresight exercises. Scientometrics, v.36, n.3, p.397-420, 1996.

ROCHA, T. V.; BORINI, F. M; SPERS, E. E.; OGASAVARA, M. H.; KHAUAJA, D.; CAMARGO, A.; MELLO, P. L. R.. Estratégias de Internacionalização das franquias brasileiras. São Paulo: ESPM, 2014.

ROSENBLOOM, B.. Canais de Marketing: uma visão gerencial. 8 ed. São Paulo: Cengage Learning, 2015.

RUBIN, P. H.. Theory of firm and structure of franchise contract. Journal of Law \& Economics, v.21, n.1, p.223-233, 1978.

VANTI, N. A. P.. Da bibliometria à webometria: uma exploração conceitual dos mecanismos utilizados para medir o registro da informação e a difusão do conhecimento. Ciência da Informação, v.31, n.2, p.152-162, 2002. 
VAZ, S. R. S.; CAMLOT, S.; GOMES, J. S.. The impact of the internationalization management control systems: case study of two food sector companies. Revista Contabilidade E Controladoria, v.7, n.1, p.43-63, 2015.

WALLIN, J. A.. Bibliometric methods: pitfalls and possibilities. Basic \& clinical pharmacology \& toxicology, v.97, n.5, p.261275, 2005.

WENG, Z. K.. Channel coordination and quantity discounts. Management Science, v.41, n.9, p.1509-1522, 1995.
WILLIAMSON, O. E.. Franchise bidding for natural monopolies in general and with respect to CATV. Bell Journal of Economics, v.7, n.1, p.73-104, 1976.

WINTER, S. G.; SZULANSKI, G.. Replication as strategy. Organization Science, v.12, n.6, p.730-743, 2001.

YAN, R.; WANG, K. Y.. Franchisor-franchisee supply chain cooperation: Sharing of demand forecast information in high-tech industries. Industrial Marketing Management, v.41, n.7, p.1164-1173, 2012. 